University of Texas at El Paso

ScholarWorks@UTEP

\title{
$5-2018$
}

\section{How to Take Expert Uncertainty into Account: Economic Approach Illustrated by Pavement Engineering Applications}

\author{
Edgar Daniel Rodriguez Velasquez \\ The University of Texas at El Paso, edrodriguezvelasquez@miners.utep.edu \\ Carlos M. Chang Albitres \\ The University of Texas at El Paso, cchangalbitres2@utep.edu \\ Thach N. Nguyen \\ Banking University of Ho Chi Minh City, ajeb@buh.edu.vn \\ Odgawkthisheledadditional works at: https://scholarworks.utep.edu/cs_techrep \\ J' University of Texas at El Paso, olgak@utep.edu \\ Part of the Computer Sciences Commons \\ Qtardithakmesinovich

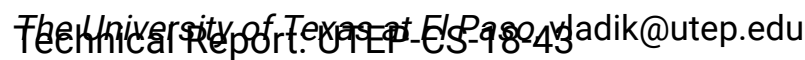

Rodriguez Velasquez E.D., Chang Albitres C.M., Nguyen T.N., Kosheleva O., Kreinovich V. (2019) How to Take Expert Uncertainty into Account: Economic Approach Illustrated by Pavement Engineering Applications. In: Kreinovich V., Sriboonchitta S. (eds) Structural Changes and their Econometric Modeling. TES 2019. Studies in Computational Intelligence, vol 808. Springer, Cham

https://doi.org/10.1007/978-3-030-04263-9_14

\section{Recommended Citation}

Rodriguez Velasquez, Edgar Daniel; Chang Albitres, Carlos M.; Nguyen, Thach N.; Kosheleva, Olga; and Kreinovich, Vladik, "How to Take Expert Uncertainty into Account: Economic Approach Illustrated by Pavement Engineering Applications" (2018). Departmental Technical Reports (CS). 1239.

https://scholarworks.utep.edu/cs_techrep/1239

This Article is brought to you for free and open access by the Computer Science at ScholarWorks@UTEP. It has been accepted for inclusion in Departmental Technical Reports (CS) by an authorized administrator of ScholarWorks@UTEP.For more information, please contact Iweber@utep.edu. 


\title{
How to Take Expert Uncertainty into Account: Economic Approach Illustrated by Pavement Engineering Applications
}

Edgar Daniel Rodriguez Velasquez, Carlos M. Chang Albitres, Thach Ngoc Nguyen, Olga Kosheleva, Vladik Kreinovich

\begin{abstract}
In many application areas, we rely on expert estimates. For example, in pavement engineering, we often rely on expert graders to gauge the condition of road segments and to see which repairs are needed. Expert estimates are imprecise; it is desirable to take the resulting uncertainty into account when making the corresponding decisions. The traditional approach is to first apply the traditional statistical methods to get the most accurate estimate and then to take the corresponding uncertainty into account when estimating the economic consequences of the resulting decision. On the example of pavement engineering applications, we show that it is beneficial to apply the economic approach from the very beginning. The resulting formulas are in good accordance with the general way how people make decisions in the presence of risk.
\end{abstract}

Edgar Daniel Rodriguez Velasquez

Department of Civil Engineering, Universidad de Piura in Peru (UDEP)

Av. Ramón Mugica 131, Piura, Peru, e-mail: edgar.rodriguez@udep.pe, and

Department of Civil Engineering, University of Texas at El Paso, $500 \mathrm{~W}$. University

El Paso, TX 79968, USA, e-mail: edrodriguezvelasquez@miners.utep.edu

Carlos M. Chang Albitres

Department of Civil Engineering, University of Texas at El Paso, $500 \mathrm{~W}$. University

El Paso, TX 79968, USA, e-mail: cchangalbitres2@utep.edu

Thach Ngoc Nguyen

Banking University of Ho Chi Minh City, 56 Hoang Dieu 2, Quan Thu Duc, Thu Duc

Ho Chi Minh City, Vietnam, e-mail: Thachnn@ buh.edu.vn

Olga Kosheleva and Vladik Kreinovich

University of Texas at El Paso, 500 W. University, El Paso, TX 79968, USA

e-mail: olgak@utep.edu,vladik@utep.edu 


\section{Formulation of the Problem}

Need for expert estimates. In many practical situations, we use experts to help make decisions.

In some cases - e.g., in medicine - we need experts because computer-based automated system are not yet able to always provide a correct diagnosis: human medical doctors are still needed.

In other case, the corresponding automatic equipment exists, but it is much cheaper to use human experts. For example, in pavement engineering, in principle, we can use automatic systems to gauge the condition of the road surface, to estimate the size of cracks and other faults, but the corresponding equipment is still reasonably expensive to use, while a human grader can make these evaluations easily. The use of human grades is explicitly mentioned in the corresponding normative documents; see, e.g., [1] (see also [5]).

Expert estimates come with uncertainty. Expert estimates usually come with uncertainty. The experts' estimates have, at best, the accuracy of about 10-15\%, up to $20 \%$; see, e.g., [3].

This observed accuracy is in the perfect accordance with the well-known "seven plus-minus two law" (see, e.g., [4, 6]), according to which a person normally divides everything into seven plus-minus two - i.e., between 5 and 9 - categories, and thus, has the accuracy between $1 / 9 \approx 10 \%$ and $1 / 5 \approx 20 \%$.

Traditional approach to dealing with this uncertainty. In the traditional approach to dealing with the expert uncertainty, we:

- first use the traditional statistical techniques to transform the expert opinion into the most accurate estimate of the desired quantity, and then

- if needed, we gauge the economic consequences of the resulting estimate.

Limitations of the traditional approach. The main limitation of the traditional approach is that while our ultimate objective is economic - how to best maintain the pavement within the given budget - we do not take this objective into account when transforming expert's opinion into a numerical estimate.

What we do in this paper. In this paper, we show how to take economic factors into account when producing the estimate. The resulting formulas are in line with the usual way how decision makers take risk into account.

\section{Traditional Approach to Transforming Expert Opinion into a Numerical Estimate: a Brief Reminder}

Main idea. An expert may describe his or her opinion in terms of a word from natural language, or by providing a numerical estimate. For each such opinion be it a word or a numerical estimate - we can find all the cases when this expert 
expressed this particular opinion, and in all these cases, find the actual value of the estimated quantity $q$.

As a result, for each opinion, we get a probability distribution on the set of all possible values of the corresponding quantity. This distribution can be described either in terms of the corresponding probability density function (pdf) $\rho(x)$, or in the terms of the cumulative distribution function (cdf) $F(x) \stackrel{\text { def }}{=} \operatorname{Prob}(q \leq x)$.

In many real-life situations, the expert uncertainty is a joint effect of many different independent factors, each of which may be small by itself. In such cases, we can take into account the Central Limit Theorem, according to which the distribution of the sum of a large number of small independent random variables is close to Gaussian (normal); see, e.g., [7]. Thus, it often makes sense to assume that the corresponding probability distribution is normal. For the normal distribution with mean $\mu$ and standard deviation $\sigma$, we have

$$
F(x)=F_{0}\left(\frac{x-\mu}{\sigma}\right),
$$

where $F_{0}(x)$ is the cdf of the standard normal distribution - with mean 0 and standard deviation 1.

Based on the probability distribution, we describe the most accurate numerical estimate.

Details: how to transform the probability distribution reflecting the expert opinion into a numerical estimate. We want to have an estimate which is as close to the actual values of the quantity $q$ as possible.

For the same opinion of an expert, we have, in general, different actual values $q_{1}, \ldots, q_{n}$. These values form a point $\left(q_{1}, \ldots, q_{n}\right)$ in the corresponding $n$ dimensional space. Once we select a numerical value $x_{0}$ corresponding to this opinion, we will generate the value $x_{0}$ in all the cases in which the experts has this particular opinion. In other words, what we generate is the point $\left(x_{0}, \ldots, x_{0}\right)$.

A natural idea is to select the estimate $x_{0}$ for which the point $\left(x_{0}, \ldots, x_{0}\right)$ is the closest to the point $\left(q_{1}, \ldots, q_{n}\right)$ that describes the actual values of the corresponding quantity. In other words, we want to select the estimate $x_{0}$ for which the distance

$$
d \stackrel{\text { def }}{=} \sqrt{\left(x_{0}-q_{1}\right)^{2}+\ldots+\left(x_{0}-q_{n}\right)^{2}}
$$

is the smallest possible.

Minimizing the distance is equivalent to minimizing its square

$$
d^{2}=\left(x_{0}-q_{1}\right)^{2}+\ldots+\left(x_{0}-q_{n}\right)^{2} .
$$

Differentiating the expression for $d^{2}$ with respect to $x_{0}$ and equating the derivative to 0 , we conclude that

$$
2\left(x_{0}-q_{1}\right)+\ldots+2\left(x_{0}-q_{n}\right)=0 .
$$


If we divide both sides of this equality by 2 , move all the terms not related to $x_{0}$ to the right-hand side, and then divide both sides by $n$, we conclude that

$$
x_{0}=\mu \text {, }
$$

where $\mu$ denotes the sample mean:

$$
\mu \stackrel{\text { def }}{=} \frac{q_{1}+\ldots+q_{n}}{n} .
$$

In terms of the probability distribution, this is equivalent to minimizing the mean square value $\int\left(x-x_{0}\right)^{2} \cdot \rho(x) d x$, which leads to $x_{0}=\mu=\int x \cdot \rho(x) d x$.

\section{How to Estimate the Economic Consequences of Selecting an Estimate: on the Example of Pavement Engineering}

Analysis of the problem: possible faults and how much it costs to repair them. In pavement engineering, we are interested in estimating the pavement fault index $x$. When the pavement is perfect, this index is 0 . The presence of any specific fault increases the value of this index.

Repairing a fault takes money; the larger the index, the more costly it is to repair this road segment. Let us denote the cost of repairs for a road segment with index $x$ by $c(x)$.

We are interested in the case when the road is regularly repaired. In this case, the index $x$ cannot grow too much - once there are some faults in the road, these faults are being repaired. Thus, the values of the index $x$ remain small. So, we can expand the unknown function into Taylor series and keep only the first terms in this expansion - e.g., only linear terms:

$$
c(x) \approx c_{0}+c_{1} \cdot x .
$$

When the road segment is perfect, i.e., when $x=0$, no repairs are needed, so the cost is $0: c(0)=0$. Thus, $c_{0}=0$, and the cost of repairs linearly depends on the index:

$$
c(x) \approx c_{1} \cdot x
$$

What is the cost of not repairing a road segment? If we do not repair a faulty road segment, then, because of the constant traffic load, in the next year, the pavement condition will become worse.

Each fault worsens. Thus, the more faults we have now, the worse will be the situation next year. Let $g(x)$ denote the next-year index corresponding to the situation when this year, the index is $x$. 
Since, as we have mentioned, it makes sense to consider small values of $x$, we can safely expand the function $g(x)$ in Taylor series and keep only linear terms in this expansion:

$$
g(x) \approx g_{0}+g_{1} \cdot x .
$$

When the pavement is perfect, i.e., when $x=0$, we usually do not expect it to deteriorate next year, so we should have $g(0)=0$. Thus, we have $g_{0}=0$, and $g(x) \approx g_{1} \cdot x$.

Since we did not repair the road segment this year, we have to repair it next year. Next year, the index will increase from the original value $x$ to the new value $x^{\prime} \stackrel{\text { def }}{=} g_{1} \cdot x$. Thus, the cost of repairs will be $c_{1} \cdot x^{\prime}=c_{1} \cdot g_{1} \cdot x$.

This is the cost next year, so to compare it with the cost of this-year repairs, we need to take into account that next year's money is somewhat cheaper than this year's money: if the interest rate is $r$, we can invest a smaller amount

$$
\frac{c_{1} \cdot q_{1} \cdot x}{1+r}
$$

now, and get the desired amount $c_{1} \cdot g_{1} \cdot x$ next year. This formula (2) describes the equivalent this-year cost of not repairing the road segment this year.

Combining these costs: what is the economic consequence of selecting an estimate. Once we select an estimate $x_{0}$ describing the quality of the road segment, we perform the repairs corresponding to this degree. According to the formula (1), these repairs costs us the amount $c_{1} \cdot x_{0}$.

If the actual value $x$ is exactly equal to $x_{0}$, this is the ideal situation: the road segment is repaired, and we spend exactly the amount of the money needed to repair it. Realistically, the actual $x$ is, in general, somewhat different from $x_{0}$. As a result, we waste some resources.

When the actual value $x$ of the pavement quality is smaller than $x_{0}$, this means that we spend too much money on repairs: e.g., we bring on heavy and expensive equipment while a simple device would have been sufficient. We could spend just $c_{1} \cdot x$ and instead, we spend a larger amount $c_{1} \cdot x_{0}$. Thus, in comparison with the ideal situation, we waste the amount

$$
c_{1} \cdot x_{0}-c_{1} \cdot x=c_{1} \cdot\left(x_{0}-x\right)
$$

When the actual value $x$ of the pavement index is larger than the estimate $x_{0}$, this means that after performing the repairs corresponding to the value $x_{0}$, we still have the remaining fault level $x-x_{0}$ which needs to be repaired next year. The cost of these repairs - when translated into this year's costs - can be found by applying the formula (2): it is

$$
\frac{c_{1} \cdot q_{1} \cdot\left(x-x_{0}\right)}{1+r}
$$

The formulas (3) and (4) describe what will be the wasted amount for each $x$. By multiplying this amount by $\rho(x)$ and integrating over $x$, we get the following expression for the expected value of the waste: 


$$
W\left(x_{0}\right) \stackrel{\text { def }}{=} \int_{0}^{x_{0}} c_{1} \cdot\left(x_{0}-x\right) \cdot \rho(x) d x+\int_{x_{0}}^{\infty} \frac{c_{1} \cdot q_{1} \cdot\left(x-x_{0}\right)}{1+r} \cdot \rho(x) d x .
$$

\section{Towards Economically Optimal Estimates}

Main idea. Instead of selecting the statistically optimal estimate

$$
x_{0}=\mu=\int x \cdot \rho(x) d x
$$

and gauging the expected waste related to this estimate, let us instead use the estimate that minimizes the waste (5).

Analysis of the problem. To find the value $x_{0}$ that minimizes the expression (5), let us differentiate this expression with respect to $x_{0}$ and equate the derivative to 0 .

The expression (5) is the sum of two terms, so the derivative of the expression (5) is equal to sum of the derivatives of these two terms.

To find the derivative of the first term, it is convenient to introduce an auxiliary function

$$
G\left(t, x_{0}\right) \stackrel{\text { def }}{=} \int_{0}^{t} c_{1} \cdot\left(x_{0}-x\right) \cdot \rho(x) d x
$$

In terms of this auxiliary function, the first term has the form $G\left(x_{0}, x_{0}\right)$. Thus, by the chain rule, the derivative of the first term can be described as

$$
\frac{d}{d x_{0}} G\left(x_{0}, x_{0}\right)={\frac{\partial G\left(t, x_{0}\right)}{\partial t}}_{\mid t=x_{0}}+{\frac{\partial G\left(t, x_{0}\right)}{\partial x_{0}}}_{\mid t=x_{0}} .
$$

It is known that differentiation and integration are inverse operations. Since (6) is an integral of some expression from 0 to $t$, its derivative with respect to $t$ is simply the value of the integrated expression for $x=t$ :

$$
\frac{\partial G\left(t, x_{0}\right)}{\partial t}=c_{1} \cdot\left(x_{0}-t\right) \cdot \rho(t)
$$

For $t=x_{0}$, this expression is equal to 0 .

The second expression in the right-hand side of the formula (7) is an integral. The derivative of the integral (i.e., in effect, of the weighted sum) is thus equal to the integral (i.e., to the weighted sum) of the corresponding derivatives:

$$
\frac{\partial G\left(t, x_{0}\right)}{\partial x_{0}}=\frac{\partial}{\partial x_{0}} \int_{0}^{t} c_{1} \cdot\left(x_{0}-x\right) \cdot \rho(x) d x=\int_{0}^{t} \frac{\partial}{\partial x_{0}}\left(c_{1} \cdot\left(x_{0}-x\right) \cdot \rho(x)\right) d x .
$$

The derivative of a linear function is simply the coefficient at the unknown $x_{0}$ :

$$
\frac{\partial}{\partial x_{0}}\left(c_{1} \cdot\left(x_{0}-x\right) \cdot \rho(x)\right)=c_{1} \cdot \rho(x)
$$


thus the expression (8) takes the form

$$
\frac{\partial G\left(t, x_{0}\right)}{\partial x_{0}}=\int_{0}^{t} c_{1} \cdot \rho(x) d x=c_{1} \cdot \int_{0}^{t} \rho(x) d x .
$$

The integral in the right-hand side of this formula is simply the value of the $\operatorname{cdf} F(t)$. So, for $t=x_{0}$, it takes the form $F\left(x_{0}\right)$. Thus:

$$
\frac{d}{x_{0}} G\left(x_{0}, x_{0}\right)=\frac{\partial G\left(t, x_{0}\right)}{\partial x_{0}}{ }_{\mid t=x_{0}}=c_{1} \cdot F\left(x_{0}\right) .
$$

To find the derivative of the second term in the right-hand side of the formula (5), let us introduce another auxiliary function

$$
H\left(t, x_{0}\right) \stackrel{\text { def }}{=} \int_{t}^{\infty} \frac{c_{1} \cdot q_{1} \cdot\left(x-x_{0}\right)}{1+r} \cdot \rho(x) d x .
$$

In terms of this auxiliary function, the second term in the expression (5) for the waset function $W\left(x_{0}\right)$ has the form $H\left(x_{0}, x_{0}\right)$. Thus, by the chain rule, the derivative of the first term can be described as

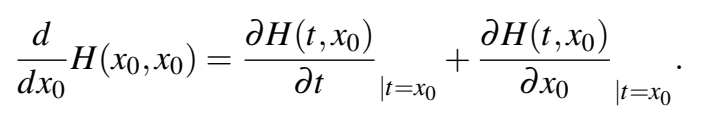

Since (10) is an integral of some expression from $t$ to $\infty$, its derivative with respect to $t$ is simply minus the integrated expression:

$$
\frac{\partial H\left(t, x_{0}\right)}{\partial t}=-\frac{c_{1} \cdot q_{1} \cdot\left(t-x_{0}\right)}{1+r} \cdot \rho(t) .
$$

For $t=x_{0}$, this expression is equal to 0 .

For the second term in the right-hand side of the formula (11), the derivative of the integral (i.e., in effect, of the weighted sum) is equal to the integral (i.e., to the weighted sum) of the corresponding derivatives:

$$
\begin{gathered}
\frac{\partial H\left(t, x_{0}\right)}{\partial x_{0}}=\frac{\partial}{\partial x_{0}} \int_{t}^{\infty} \frac{c_{1} \cdot q_{1} \cdot\left(x-x_{0}\right)}{1+r} \cdot \rho(x) d x= \\
\int_{t}^{\infty} \frac{\partial}{\partial x_{0}}\left(\frac{c_{1} \cdot q_{1} \cdot\left(x-x_{0}\right)}{1+r} \cdot \rho(x)\right) d x .
\end{gathered}
$$

The derivative of a linear function is simply the coefficient at the unknown $x_{0}$ :

$$
\frac{\partial}{\partial x_{0}}\left(\frac{c_{1} \cdot q_{1} \cdot\left(x-x_{0}\right)}{1+r} \cdot \rho(x)\right)=\frac{c_{1} \cdot q_{1}}{1+r} \cdot \rho(x),
$$

thus the expression (12) takes the form 


$$
\frac{\partial H\left(t, x_{0}\right)}{\partial x_{0}}=\int_{t}^{\infty} \frac{c_{1} \cdot q_{1}}{1+r} \cdot \rho(x) d x=\frac{c_{1} \cdot q_{1}}{1+r} \cdot \int_{t}^{\infty} \rho(x) d x .
$$

The integral in the right-hand side of this formula is simply the 1 minus value of the cdf $F(t)$. So for $t=x_{0}$, it takes the form $1-F\left(x_{0}\right)$. Thus, this derivative takes the following form:

$$
{\frac{\partial H\left(t, x_{0}\right)}{\partial t}}_{\mid t=x_{0}}=\frac{c_{1} \cdot q_{1}}{1+r} \cdot\left(1-F\left(x_{0}\right)\right) .
$$

As we have mentioned, the derivative of the objective function (5) - the derivative which should be equal to 0 when we select the economically optimal estimate $x_{0}-$ is equal to the sum of the expressions (9) and (13). Thus, the optimality condition $\frac{d W\left(x_{0}\right)}{d x_{0}}=0$ takes the form

$$
c_{1} \cdot F\left(x_{0}\right)-\frac{c_{1} \cdot q_{1}}{1+r} \cdot\left(1-F\left(x_{0}\right)\right)=0
$$

If we divide both sides of this equality by $c_{1}$, move all the terms not containing the unknown $F\left(x_{0}\right)$ to the right-hand side, and divide by the coefficient at $F\left(x_{0}\right)$, we conclude that

$$
F\left(x_{0}\right)=\frac{\frac{q_{1}}{1+r}}{1+\frac{q_{1}}{1+r}}=\frac{q_{1}}{1+r+q_{1}} .
$$

Main conclusion. As the estimate corresponding to the expert's opinion, we should select not the mean of the actual values corresponding to this opinion, but rather a quantile corresponding to the level $\frac{q_{1}}{1+r+q_{1}}$ :

$$
F\left(x_{0}\right)=\frac{q_{1}}{1+r+q_{1}},
$$

where:

- $q_{1}$ is the growth rate of the pavement fault - what fault of index 1 will grow into next year, and

- $r$ is the interest rate - how much interest we will get if we invest \$1 now.

Discussion. If the fault growth is negligible, i.e., if $q_{1} \approx 1$, then, taking into account that $r$ is very small, we conclude that $F\left(x_{0}\right) \approx 1 / 2$, i.e., $x_{0}$ should be the median of the corresponding probability distribution.

For symmetric distributions like normal, median and mean coincide - they both coincide with the center of the distribution, i.e., with the value with respect to which this distribution is symmetric. In this case, we can still use the statistically optimal estimate $x_{0}=\mu$. 
However, in most real-life situations, when $q_{1} \gg 1+r$, we have $\frac{1+r}{q_{1}} \ll 1$, thus, $1+\frac{1+r}{q_{1}} \ll 2$ and

$$
F\left(x_{0}\right)=\frac{q_{1}}{1+r+q_{1}}=\frac{1}{1+\frac{1+r}{q_{1}}} \gg 0.5,
$$

so we should select the values larger than the mean.

For the case of the normal distribution, with $F(x)=F_{0}\left(\frac{x-\mu}{\sigma}\right)$, the formula (14) takes the form

$$
F_{0}\left(\frac{x-\mu}{\sigma}\right)=\frac{q_{1}}{1+r+q_{1}}
$$

i.e., the form

$$
\frac{x-\mu}{\sigma}=k,
$$

where $k$ is the value for which

$$
F_{0}(k)=\frac{q_{1}}{1+r+q_{1}} .
$$

Thus, instead of the statistically optimal estimate $x_{0}=\mu$, we need to use the estimate

$$
x_{0}=\mu+k \cdot \sigma .
$$

This is in line with the usual way to taking risk into account when comparing different alternatives: instead of comparing average gains $\mu$, we should compare the values $\mu-k \cdot \sigma$, where the coefficient $k$ depends on the person's tolerance to risk; see, e.g., [2] and references therein.

Comment. We recommend plus $k \cdot \sigma$, since instead of maximizing gains, we minimize losses - i.e., negative gains. When we switch from the value to negative of this value, then $\mu+k \cdot \sigma$ becomes $\mu-k \cdot \sigma$. Indeed, $\mu[-x]=-\mu[x]$, while $\sigma[-x]=\sigma[x]$, so

$$
\mu[-x]+k \cdot \sigma[-x]=-\mu[x]+k \cdot \sigma[x]=-(\mu[x]-k \cdot \sigma[x]) .
$$

Practical recommendation for pavement engineering (and for other similar applications). For each expert opinion, we collect all the cases in which the expert expressed this opinion, and find, in all these cases, the actual values of the corresponding quantity. Based on these actual values, we compute the mean $\mu$ and the standard deviation $\sigma$. Then, as a numerical description of the expert's opinion, we select the value $\mu+k \cdot \sigma$, where $k$ is determined by the formula (16).

This way, we can decrease the losses caused by the expert's uncertainty. 


\section{Acknowledgments}

This work was supported in part by the US National Science Foundation grant HRD1242122 (Cyber-ShARE Center).

\section{References}

1. ASTM International, Standard Practice for Roads and Parking Lots Pavement Condition Index Surveys, International Standard D6433-18.

2. E. J. Elton, M. J. Gruber, S. J. Brown, and W. N. Goetzman, Modern Portfolio Theory and Investment Analysis, Wiley, New York, 2014.

3. Metropolitan Transportation Commission (MTC), MTC Rater Certification Exam, Streetsaver Academy, San Francisco, California, 2018.

4. G. A. Miller, "The magical number seven, plus or minus two: Some limits on our capacity for processing information", Psychological Review, 1956, Vol. 63, No. 2, pp. 81-97.

5. K. Park, N. E. Thomas, and K. W. Lee, "Applicability of the International Roughness Index as a predictor of asphalt pavement condition”, Journal of Transportation Engineering, 2007, Vol. 133, No. 12, pp. 706-709.

6. S. K. Reed, Cognition: Theories and Application, Wadsworth Cengage Learning, Belmont, California, 2010.

7. D. J. Sheskin, Handbook of Parametric and Nonparametric Statistical Procedures, Chapman and Hall/CRC, Boca Raton, Florida, 2011. 